\title{
FoxA2 hunting research identifies the early trail of mesenchymal differentiation
}

\author{
Paolo Madeddu
}

See related research by Howard et al., http://stemcellres.com/content/4/2/36

\begin{abstract}
Epigenetic regulation offers a flexible means to instruct cell functions and fate. In human embryonic stem cells (hESCs), thousands of genes are targets for histone modifications leading to activation or suppression of transcription. Novel research now indicates that, in $\mathrm{hESCS}$, the transcription start site of FOXA2, encoding a member of the forkhead family of transcription factors, is bivalently marked with histone modifications for both gene activation and repression. Moreover, FOXA2 is remarkably upregulated at an early stage of endothelial differentiation. These discoveries provide better understanding of the natural program of differentiation and also open up new opportunities for large scale production of endothelial progenitors.
\end{abstract}

Scientists are struggling to dissect the mechanisms underlying the specification of pluripotent cells, that is, embryonic stem cells (ESCs), into different cell lineages. Even more challenging is the task of pushing ESCs into specific lineages and thereby developing directed differentiation protocols, ultimately increasing the yield of vascular cells for regenerative medicine therapies in cardiovascular disease.

Howard and colleagues newly investigate the expressional and epigenetic changes incurred by human ESCs (hESCs) during directed differentiation to endothelial cells, by employing gene microarrays, quantitative RT-PCR, immunocytochemistry, and chromatin immunoprecipitation (ChIP) analysis [1]. Using a feeder- and serum-free differentiation protocol previously set up for derivation of endothelial cells $[2,3]$, they show that 22 transcription factors are specific to early mesoderm commitment. Among

Correspondence: madeddu@yahoo.com

Chair of Experimental Cardiovascular Medicine, Section of Regenerative Medicine, School of Clinical Sciences, University of Bristol, Bristol BS2 8HW, United Kingdom these, FOXA2 emerged as the most significantly differentially expressed at the day 2 time point. FOXA2, also known as hepatocyte nuclear factor 3-beta (HNF-3B), belongs to the $\mathrm{O}$ subclass of the forkhead family of transcription factors, which are characterized by a distinct forkhead DNA-binding domain. In particular, FOXA2 acts as a transcriptional activator for liver-specific genes such as those encoding albumin and transthyretin and also interacts with chromatin to produce post-translational modification of histones.

Our current knowledge of the regulation of epigenetic processes in stem cells is still very limited, but new evidence highlights their association with changes in the structure of chromatin and DNA. Epigenetic mechanisms can induce plastic, short-term modification on chromatin structure by histone tail modifications, as well as rigid, long-term effects by DNA methylation. Modified histone residues are able to 'cross-talk' among each other, thus constituting the docking site for distinct and specific chromatin-binding proteins that direct the dynamic transition between transcriptionally active and silent chromatin [4]. Importantly, thousands of developmental regulatory genes in hESCs share methylation targets that are associated with gene activation or repression [5], allowing them to be rapidly switched on/off upon differentiation into alternative cell lineages. Using ChIP-PCR analysis, Howard and colleagues show that the FOXA2 transcription start site is bivalently marked with histone modifications for both gene activation (H3K4me3) and repression (H3K27me3) in hESCs, thus suggesting that the transcription factor may be a key regulator of hESC differentiation. It is worth noting that FOXA2 is upregulated at the early stage of hESC differentiation and therefore it can represent a primordial regulator of lineage commitment. Nevertheless, further studies are guaranteed to confirm that harnessing FOXA2 could be utilitarian to generation of desired regenerative cells, such as endothelial progenitors or pericytes. In addition, it is of paramount importance to extend this approach to the investigation of cardiovascular 
development to verify if FOXA2 plays a role in the regulation of mesoderm specification and epithelial-mesenchymal transition [6]. Furthermore, these early developmental programs might be re-activated in the adult heart and vasculature after injury and play a role in the regeneration/repair of the cardiovascular system. Thus, hunting FOXA2 might be crucial not only for designing directed endothelial differentiation protocols but also for directly encouraging intrinsic mechanisms of repair. In this context, the article of Howard and colleagues is a horn call blowing for the pack to start the hunt.

\section{Abbreviations}

ChIP: Chromatin immunoprecipitation; ESC: Embryonic stem cell; hESC: Human embryonic stem cell; RT-PCR: Real-time polymerase chain reaction.

\section{Competing interests}

The author declares that they have no competing interests.

Published: 24 April 2013

\section{References}

1. Howard L, Mackenzie RM, Pchelintsev NA, McBryan T, McClure JD, McBride MW, Kane NM, Adams PD, Milligan G, Baker AH: Profiling of transcriptional and epigenetic changes during directed endothelial differentiation of human embryonic stem cells identifies FOXA2 as a marker of early mesoderm commitment. Stem Cell Res Ther 2013, 4:36.

2. Kane NM, Meloni M, Spencer HL, Craig MA, Strehl R, Milligan G, Houslay MD, Mountford JC, Emanueli C, Baker AH: Derivation of endothelial cells from human embryonic stem cells by directed differentiation: analysis of microRNA and angiogenesis in vitro and in vivo. Arterioscler Thromb Vasc Biol 2010, 30:1389-1397.

3. Kane NM, Howard L, Descamps B, Meloni M, McClure J, Lu R, McCahill A Breen C, Mackenzie RM, Delles C, Mountford JC, Milligan G, Emanueli C, Baker AH: Role of microRNAs 99b, 181a, and 181b in the differentiation of human embryonic stem cells to vascular endothelial cells. Stem Cells 2012, 30:643-654.

4. Kouzarides T: Chromatin modifications and their function. Cell 2007, 128:693-705.

5. Bernstein BE, Mikkelsen TS, Xie X, Kamal M, Huebert DJ, Cuff J, Fry B, Meissner A, Wernig M, Plath K, Jaenisch R, Wagschal A, Feil R, Schreiber SL, Lander ES: A bivalent chromatin structure marks key developmental genes in embryonic stem cells. Cell 2006, 125:315-326.

6. Nakajima $Y$, Imanaka-Yoshida K: New insights into the developmental mechanisms of coronary vessels and epicardium. Int Rev Cell Mol Biol 2013, 303:263-317

\section{doi:10.1186/scrt188}

Cite this article as: Madeddu: FoxA2 hunting research identifies the early trail of mesenchymal differentiation. Stem Cell Research \& Therapy 2013 4:40. 